\title{
Uso de corticoides sistémicos no tratamento da artrite gotosa aguda: qual a evidência?
}

Ana Margarida Costa, ${ }^{1}$ Ana Isabel Ribeiro da Silva ${ }^{2}$

\section{RESUMO}

Objetivo: Avaliar a eficácia e segurança dos corticoides sistémicos (CS) no tratamento da artrite gotosa aguda (AGA) e os seus benefícios face às terapêuticas clássicas nomeadamente a colchicina e os anti-inflamatórios não esteroides (AINEs).

Fontes de dados: MEDLINE e sítios de medicina baseada na evidência.

Métodos de revisão: Pesquisa de normas de orientação clínica (NOC), revisões sistemáticas (RS), ensaios clínicos aleatorizados e controlados (ECAC) e meta-análises (MA), publicados entre junho/2007 e junho/2017, utilizando os termos MeSH glucocorticoids, gout suppressants, adrenal cortex hormones, acute disease, gout e hyperuricemia. Para avaliar o nível de evidência foi aplicada a escala Strenght of Recommendation Taxonomy (SORT), da American Family Physician.

Resultados: Obtiveram-se 731 artigos, selecionando-se oito NOC, duas RS e dois ECAC. As NOC referem melhoria da sintomatologia da AGA com o uso de CS, AINEs ou colchicina, divergindo nas recomendações relativamente à terapêutica de primeira linha mais adequada. Se, por um lado, alguns estudos mencionam uma melhoria mais significativa da sintomatologia com o uso de CS, principalmente nos primeiros dias de tratamento, por outro lado, citam-nos como tão eficazes quanto o tratamento clássico. De uma forma geral, todos referem mais efeitos secundários nos doentes tratados com AINEs em comparação com os CS.

Conclusões: Os CS, os AINEs e a colchicina são todos eficazes na gestão terapêutica da AGA. No entanto, os CS apresentam superioridade relativamente aos AINES no que respeita à segurança. Desta forma, a escolha do agente para o tratamento da AGA deve ser determinada com base no perfil do doente.

Palavras-chave: Gota; Corticoides.

\section{INTRODUÇÃO}

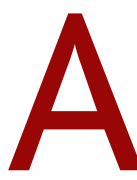
gota é uma das artropatias inflamatórias mais comuns na Europa, cuja prevalência, em aumento contínuo nos últimos anos, varia de 0,9 a 2,5\%. ${ }^{1}$ A artrite gotosa aguda (AGA) é caracterizada por episódios agudos intermitentes de sinovite, secundários à deposição articular de cristais de urato monossódico. Estes episódios caracterizam-se por edema e dor articular, cuja frequência, severidade e duração se intensificam com a progressão da doença. Alguns doentes desenvolvem uma condição inflamatória crónica, associada habitualmente a tofos gotosos.

1. Médica Interna de Medicina Geral e Familiar. USF Physis, ACeS Alto Ave 2. Médica Interna de Medicina Geral e Familiar. USF Pevidém, ACeS Alto Ave
Segundo as últimas recomendações da Sociedade Portuguesa de Reumatologia (SPR), na suspeita clínica de AGA a identificação de depósitos de uratos é sempre desejável. ${ }^{2}$

Embora o tratamento precoce tenha contribuído para a melhoria do seu prognóstico, trata-se de uma entidade clínica subdiagnosticada e com gestão sub-ótima numa grande proporção de doentes. ${ }^{1}$

A abordagem terapêutica da AGA, no que respeita à escolha de primeira linha, é controversa. Existem argumentos para reavaliar o status dos anti-inflamatórios não esteroides (AINEs) e da colchicina como tratamento padrão, devido às respetivas contraindicações e efeitos adversos associados, pelo que é necessário avaliar alternativas com melhor perfil terapêutico. ${ }^{3}$

Neste sentido, os autores pretendem rever a evidência mais recente sobre a eficácia e a segurança dos corticoides sistémicos (CS) no tratamento da AGA, clarifi- 
cando qual a terapêutica de primeira linha mais adequada, com principal destaque na comparação entre colchicina/AINEs e CS.

\section{MÉTODOS}

Foi realizada uma pesquisa de normas de orientação clínica (NOC), revisões sistemáticas (RS), ensaios clínicos aleatorizados e controlados (ECAC) e meta-análises (MA) publicados entre junho/2007 e junho/2017, nas línguas portuguesa e inglesa, indexados nas bases de dados da National Guideline Clearinghouse, The Cochrane Library, Database of Abstracts of Reviews of Effectiveness (DARE), TRIP e MEDLINE, utilizando os termos MeSH: glucocorticoids; gout suppressants; adrenal cortex hormones; acute disease; gout; hyperuricemia.

Os critérios utilizados para a inclusão dos artigos consistiram em: população-alvo constituída por adultos com AGA; intervenção terapêutica com CS em comparação com colchicina ou AINEs; outcome primário com avaliação da intensidade da dor e outcome secundário com a ocorrência de eventos adversos.

Foram utilizados como critérios de exclusão: estudos não randomizados, artigos duplicados, artigos de opinião, artigos editoriais e artigos discordantes do objetivo da revisão. Foram também excluídos ensaios clínicos incluídos em RS ou MA selecionadas e artigos escritos pelo mesmo autor que sofreram posteriores atualizações.

Para avaliar o nível de evidência (NE) dos estudos e a força de recomendação (FR) foi aplicada a escala Strenght of Recommendation Taxonomy (SORT), da American Academy of Family Physicians. ${ }^{4}$

A seleção dos artigos para revisão foi feita em duplicado pelos dois autores que, em caso de dúvidas, perante inclusão do título, deliberaram incluir o artigo para leitura integral. A inclusão/exclusão do artigo após leitura integral foi realizada pelos dois autores em simultâneo, com concordância absoluta.

\section{RESULTADOS}

A pesquisa efetuada resultou na identificação de 731 artigos e na seleção de 12 que respeitavam todos os critérios de inclusão e de exclusão: oito NOC, duas RS e dois ECAC. A Figura 1 representa o organigrama da seleção dos artigos. Os Quadros I, II e III resumem as características dos estudos selecionados para a revisão.

As NOC do American College of Rheumatology (ACR) ${ }^{5}$ e da European League Against Rheumatism (EULAR), ${ }^{1}$ assim como as NOC multinacionais, ${ }^{6-7}$ recomendam na abordagem da AGA o uso de terapêutica farmacológica em monoterapia com AINEs, CS ou colchicina oral em baixas doses, sem estabelecer prioridade entre essas opções (FR A). A EULAR ressalva que o tratamento deverá ser iniciado o mais precocemente possível (preferencialmente nas primeiras 24 horas após o início da crise) e baseado na preferência do doente, resposta prévia à terapêutica farmacológica em situação de crise, comorbilidades associadas e no número e tipo de articulações envolvidas (FR C). Reforça ainda que a colchicina e os AINEs devem ser evitados em pacientes com insuficiência renal severa (FR C) e a colchicina não deve ser administrada em pacientes medicados com inibidores CYP3A4 e glicoproteína-P, como a ciclosporina e claritromicina (FR B). A terapêutica combinada, mencionada pelo ACR, é uma opção apropriada quando a crise é caracterizada por dor intensa e em artrites poliarticulares ou crises envolvendo mais de duas grandes articulações (FR C).

De acordo com as recomendações publicadas pela British Society for Rheumatology $y^{3}$ e pela SPR, ${ }^{2}$ os AINEs na dose máxima ou a colchicina são os fármacos de escolha para a AGA, sendo os CS opções alternativas na presença de contraindicações (FR B). Esta recomendação mostra-se inconsistente uma vez que se apoia numa RS da Cochrane, que incluiu um ensaio randomizado duplamente cego que considera os CS igualmente eficazes aos AINEs e não uma alternativa.

As NOC da Sociedad Española de Reumatologia ${ }^{9}$ apontam os AINEs como a primeira opção terapêutica (FR B), acrescentando que os inibidores da ciclooxigenase (COXIBs) podem ser considerados em doentes de médio ou alto risco gastrointestinal (FR B). Por outro lado, os CS ficam reservados para os pacientes com contraindicações aos AINEs (FR B) e a colchicina em baixas doses é recomendada apenas se iniciada nas $24 \mathrm{~h}$ iniciais da crise aguda da gota (FR B).

Recentemente, o American College of Physicians ${ }^{10} \mathrm{co}-$ loca os CS como tratamento de primeira linha em pacientes sem contraindicações, alegando que são geralmente mais seguros, por apresentarem menos efeitos adversos gastrointestinais que os AINEs e uma alternativa de menor custo (FR B).

A RS conduzida por Underwood ${ }^{11}$ incluiu dois ECAC, cujo objetivo consistia na avaliação do benefício do uso 


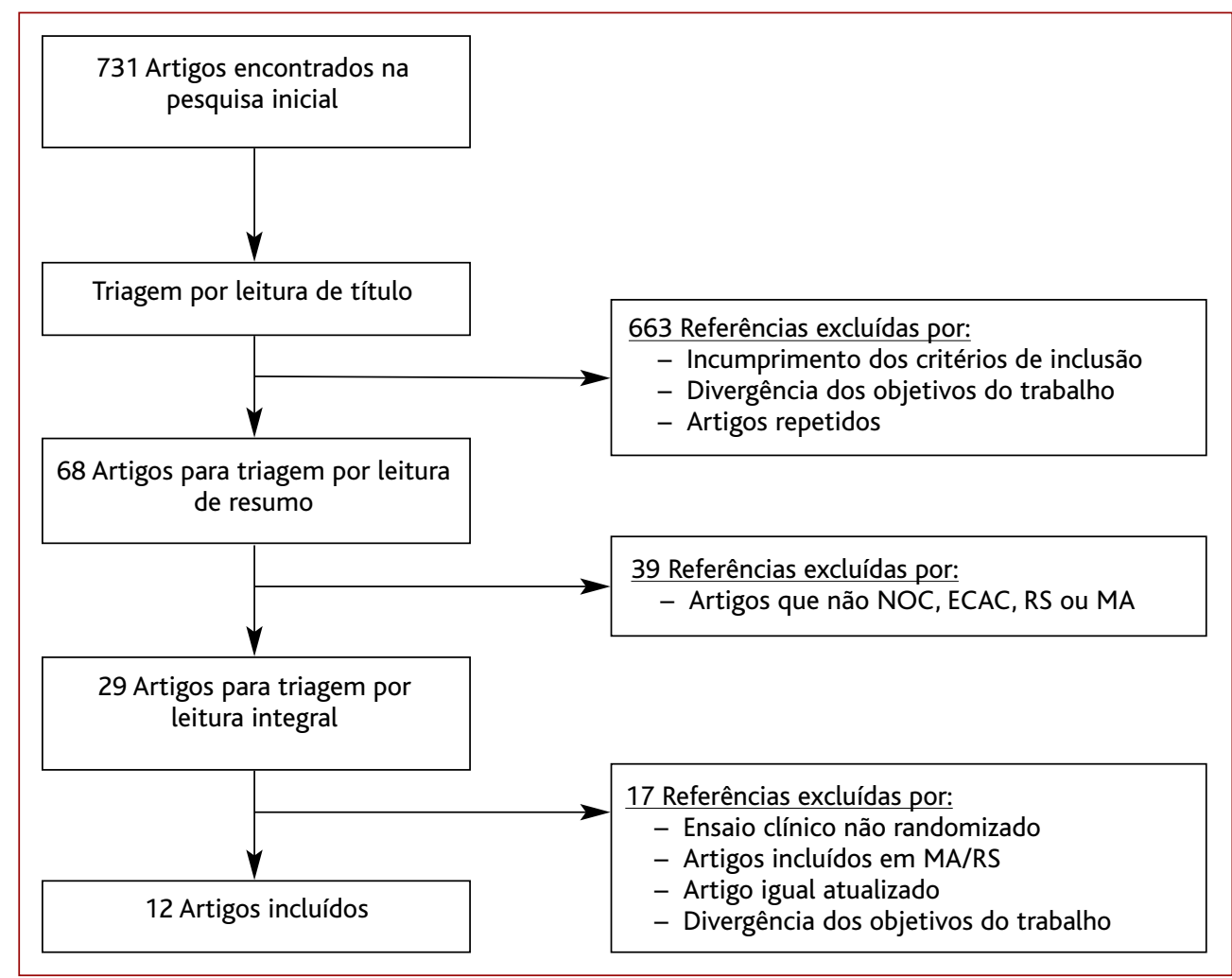

Figura 1. Organigrama da seleção dos artigos obtidos.

Legenda: $\mathrm{NOC}$ = norma de orientação clínica; $\mathrm{ECAC}$ = ensaio clínico aleatorizado e controlado; $\mathrm{RS}$ = revisão sistemática; MA = meta-análise.

deCS versusAINEs isoladamente ou em associação no tratamento da AGA. Um dos ensaios ${ }^{3,12-13}$ comparou o diclofenac associado à indometacina versus prednisolona. Para isso selecionou 90 doentes que recorreram a um serviço de urgência com sinais sugestivos de AGA, dos quais 74 eram do sexo masculino com idade média de 65 anos. $\mathrm{O}$ outcome avaliado foi a taxa de diminuição da dor [segundo a escala visual analógica (EVA)] em três momentos distintos: duas horas após a admissão, entre o dia 1 e o dia 14 e às duas semanas. A prednisolona revelou uma melhoria superior mas pouco significativa $(p=0,04)$ entre o dia 1 e o dia 14, não existindo diferenças entre os grupos nos outros momentos de avaliação. Os efeitos adversos totais foram menores nos que receberam prednisolona comparados com os que receberam indometacina $(27 \%$ versus $63 \%$, respetivamente; $p=0,0007)$. O segundo ECAC, ${ }^{12-13}$ duplamente cego, foi realizado na Holanda, durante o período de março de 2004 a julho de 2007. Os doentes incluídos foram diagnosticados com AGA, confirmada através do lí- quido sinovial da articulação afetada, sendo que a idade média foi de 57 anos e $89 \%$ eram do sexo masculino. Um grupo de doentes foi medicado com prednisolona ( $n=60)$ e outro grupo com naproxeno $(n=60)$. Não se constataram diferenças significativas entre os dois grupos na redução da dor e nos efeitos adversos $(66 \%$ versus $63 \%$, respetivamente, $p=0,42$ ). Os ECAC incluídos nesta RS cumprem critérios de boa qualidade, mas apresentam uma amostra pequena, pelo que foi atribuído NE 2.

Um dos ECAC ${ }^{14}$ incluídos numa RS realizada por Shekelle e colaboradores ${ }^{15}$ avaliou a redução da dor e a segurança da prednisolona versusindometacina. A população selecionada foi de doentes com AGA $(n=416)$, que se apresentaram em quatro serviços de urgência em Hong Kong, com menos de três dias de evolução e idade igual ou superior a 18 anos. A eficácia analgésica foi definida como melhoria da dor (em repouso ou com atividade) superior a 13mm na EVA (cotado de 1 a 100mm), tendo sido avaliada durante as primeiras duas horas e entre os dias 1 a 14. Reduções equivalentes e clinicamente significativas no score médio da dor foram observadas com a indometacina e prednisolona às duas horas [aproximadamente $10 \mathrm{~mm}$ (em repouso) e $20 \mathrm{~mm}$ (com atividade)] e nos dias 1 a 14 [aproximadamente $25 \mathrm{~mm}$ (em repouso) e $45 \mathrm{~mm}$ (com atividade)]. Eventos adversos major não ocorreram durante o estudo. No entanto, os doentes do grupo da indometacina tiveram mais eventos adversos minor comparativamente aos do grupo da prednisolona ( $19 \%$ vs. $6 \% ; p<0,001)$. Pelo facto de o ECAC incluído nesta RS apresentar uma boa qualida- 


\begin{tabular}{|c|c|c|}
\hline Referência & País & Recomendação \\
\hline $\begin{array}{l}\text { American College of } \\
\text { Rheumatology (2012) }\end{array}$ & EUA & $\begin{array}{l}\text { Para crises de severidade leve/moderada é recomendado iniciar terapêutica em } \\
\text { monoterapia com AINEs, CS ou colchicina oral (FR A). }\end{array}$ \\
\hline $\begin{array}{l}\text { European League } \\
\text { Against Rheumatism } \\
\text { (2016) }\end{array}$ & - & $\begin{array}{l}\text { A escolha do fármaco deve ser baseada no tempo decorrido após o início da crise, na } \\
\text { presença de contraindicações, experiência do paciente com tratamentos prévios e no } \\
\text { número e tipo de articulações envolvidas (FR C). } \\
\text { Para o tratamento de primeira linha usar colchicina e/ou AINEs ou CS (FR A). }\end{array}$ \\
\hline $\begin{array}{l}\text { Recomendações } \\
\text { nacionais e } \\
\text { multinacionais da } \\
\text { Iniciativa 3e (2015) }\end{array}$ & $\begin{array}{l}\text { Austrália e } \\
\text { Nova } \\
\text { Zelândia }\end{array}$ & $\begin{array}{l}\text { Os AINEs, colchicina em baixas doses e CS são todos eficazes no tratamento da AGA } \\
\text { (FR A). A escolha do agente deve ser influenciada pelas comorbilidades e medicações } \\
\text { concomitantes. }\end{array}$ \\
\hline $\begin{array}{l}\text { Recomendações } \\
\text { multinacionais da } \\
\text { Iniciativa 3e (2014) }\end{array}$ & - & $\begin{array}{l}\text { No tratamento da AGA pode usar-se colchicina em baixas doses, AINEs e/ou CS, } \\
\text { dependendo das comorbilidades e do risco de eventos adversos (FR A). }\end{array}$ \\
\hline $\begin{array}{l}\text { British Society for } \\
\text { Rheumatology (2017) }\end{array}$ & $\begin{array}{l}\text { Reino } \\
\text { Unido }\end{array}$ & $\begin{array}{l}\text { Na ausência de contraindicações, os AINEs ou a colchicina são os fármacos de escolha } \\
\text { para a AGA (FR A). } \\
\text { Os CS são alternativos nos doentes com comorbilidades ou contraindicações (FR B). }\end{array}$ \\
\hline $\begin{array}{l}\text { Sociedade Portuguesa } \\
\text { de Reumatologia } \\
(2014)\end{array}$ & Portugal & $\begin{array}{l}\text { No tratamento da AGA recomenda-se o uso de doses baixas de colchicina ou AINEs orais } \\
\text { (FR A). } \\
\text { Perante ineficácia ou contraindicação deve ser ponderado o uso de CS (FR B). }\end{array}$ \\
\hline $\begin{array}{l}\text { Sociedad Española de } \\
\text { Reumatologia (2013) }\end{array}$ & Espanha & $\begin{array}{l}\text { Os AINEs são eficazes na AGA, sendo o tratamento de primeira escolha na ausência de } \\
\text { contraindicações (FR A). } \\
\text { Os CS são recomendados para pacientes com contraindicações para AINEs (FR B). }\end{array}$ \\
\hline $\begin{array}{l}\text { American College of } \\
\text { Physicians (2017) }\end{array}$ & EUA & $\begin{array}{l}\text { Recomenda que os clínicos escolham os CS, AINEs ou colchicina para o tratamento dos } \\
\text { pacientes com AGA (FR A). } \\
\text { Os CS devem ser considerados de primeira linha em pacientes sem contraindicações, uma } \\
\text { vez que são geralmente mais seguros e uma opção de menor custo (FR B). }\end{array}$ \\
\hline
\end{tabular}

Legenda: $\mathrm{EUA}=$ Estados Unidos da América; $\mathrm{AINEs}=$ anti-inflamatórios não esteroides; $\mathrm{CS}=$ corticoides sistémicos; $\mathrm{AGA}=$ artrite gotosa aguda.

de, com uma descrição adequada da metodologia, atribuiu-se um NE 1.

Zhang e colaboradores ${ }^{16}$ publicaram em 2014 os resultados de um ECAC, realizado no Hospital Qilu da Universidade de Shandong, que decorreu de julho de 2009 a julho de 2012. Neste ensaio foram incluídos 60 pacientes com AGA com menos de 24 horas de evolução e que apresentavam dor de intensidade moderada ( 2 em 5 na escala de Likert), sendo que $97 \%$ eram do sexo masculino, com média de idades de 53 anos. Os doentes foram randomizados (1:1) para receberem betametasona ou diclofenac durante sete dias. Um dos outcomes avaliados foi a intensidade da dor. A percen- tagem de pacientes que reportou dor extrema ou severa, no início do estudo, foi de $90 \%$ no grupo que recebeu betametasona e 93,3\% no grupo que recebeu diclofenac, tendo sido reduzida para $56,7 \%$ e $73,3 \%$, respetivamente, quatro horas após iniciar o tratamento. $\mathrm{O}$ que se observou foi que a betametasona apresentou eficácia superior ao diclofenac nos primeiros três dias de tratamento $(p<0,05)$, sem diferenças significativas entre os dois grupos nos restantes dias. Para além disso, o grupo que recebeu betametasona teve menos efeitos adversos (13,3\%) que o grupo que recebeu diclofenac $(26,7 \%)$. Um outro ECAC, ${ }^{17}$ realizado na China entre abril e agosto de 2015, num departamento de en- 


\begin{tabular}{|c|c|c|c|c|}
\hline Referência & $\begin{array}{l}\text { Estudos } \\
\text { incluídos }\end{array}$ & Intervenção & Resultados & NE \\
\hline $\begin{array}{l}\text { Underwood } \\
\text { (2011) }\end{array}$ & $\begin{array}{l}2 \text { ECAC } \\
n=210\end{array}$ & $\begin{array}{l}\text { Prednisolona oral } 30 \mathrm{mg} / \text { dia, } 5 \text { dias vs } \\
\text { diclofenac } 75 \mathrm{mg} \text { IM }+ \text { indometacina oral } \\
50 \mathrm{mg} \text { tid, } 2 \text { dias, seguido de } 25 \mathrm{mg} \text { tid, } 3 \text { dias } \\
(\mathrm{n}=90) \\
\text { Prednisolona oral } 35 \mathrm{mg} / \mathrm{dia}, 5 \text { dias } v s \\
\text { naproxeno oral } 500 \mathrm{mg} \text { bid, } 5 \text { dias }(n=120 \text { ) }\end{array}$ & $\begin{array}{l}2 \text { Horas } \\
\text { Sem diferenças estatisticamente significativas } \\
\text { na redução da dor entre os dois grupos } \\
(p=0,12) \text {. } \\
\text { Dia } 1-14 \\
\text { Redução da dor estatisticamente significativa, } \\
\text { a favor da prednisolona oral ( } p=0,04) \text {. } \\
\text { Duas semanas } \\
\text { Resultados não significativos (valor } p \text { não } \\
\text { reportado) na redução da dor. } \\
\text { Os eventos adversos totais foram menores no } \\
\text { grupo da prednisolona ( } 27 \% \text { vs } 63 \% ; p=0,0007) \text {. } \\
\text { Sem diferenças estatisticamente significativas } \\
\text { na redução da dor entre os dois grupos (valor } \\
p \text { não reportado) e nos efeitos adversos ( } 66 \% \\
\text { vs } 63 \% ; p=0,42) \text {. }\end{array}$ & 1 \\
\hline $\begin{array}{l}\text { Shekelle, et } \\
\text { al. (2017) }\end{array}$ & $\begin{array}{l}1 \text { ECAC } \\
n=416\end{array}$ & $\begin{array}{l}\text { Prednisolona oral } 30 \mathrm{mg} / \mathrm{dia}, 5 \text { dias vs } \\
\text { indometacina oral } 50 \mathrm{mg} \text { tid, } 2 \text { dias, seguido } \\
\text { de } 25 \mathrm{mg} \text { tid, } 3 \text { dias }(n=416)\end{array}$ & $\begin{array}{l}\text { Sem diferenças estatisticamente significativas } \\
\text { na redução da dor. Os eventos adversos minor } \\
\text { foram menores no grupo da prednisolona } \\
\text { durante as primeiras duas horas após o início } \\
\text { do tratamento ( } 19 \% \text { vs } 6 \% ; p<0,001) \text {. }\end{array}$ & 1 \\
\hline
\end{tabular}

Legenda: $\mathrm{ECAC}=$ ensaio clínico aleatorizado controlado; $\mathrm{IM}=$ intramuscular; tid = três vezes ao dia; bid = duas vezes ao dia.

docrinologia, avaliou a eficácia da prednisolona oral, etoricoxib e indometacina no tratamento da AGA. Os doentes incluídos apresentavam idade igual ou superior a 18 anos e AGA, cuja sintomatologia tinha menos de 72 horas de evolução. Foram analisados 113 pacientes, previamente aleatorizados para receberem durante quatro dias prednisolona $(n=33)$, etoricoxib $(n=44)$ ou indometacina ( $n=36)$. O outcome primário consistiu na avaliação da redução da dor referida pelo paciente, de acordo com a escala de Likert. A prednisolona, o etoricoxib e a indometacina foram similarmente efetivos na redução da dor $(p>0,05)$. Para além disso, existiram mais efeitos adversos totais com a indometacina $(30,6 \%)$ do que com o etoricoxib $(6,8 \%)$ e a prednisolona $(6,1 \%)$ $(p=0,003)$. O facto destes dois ensaios incluírem um pequeno número de participantes pode ter conduzido a vieses significativos, pelo que lhes foi atribuído um $\mathrm{NE}$ 2.

\section{CONCLUSÕES}

Os CS, os AINEs na dose máxima e a colchicina em baixas doses são todos eficazes na gestão terapêutica da AGA (FR A). Apesar de não existirem ensaios clínicos de corticoides versus placebo no tratamento da AGA, estes têm demonstrado eficácia noutras condições inflamatórias, o que aumenta a confiança de que são igualmente efetivos no tratamento da reação inflamatória da AGA.

Em relação à preferência de uma medicação em detrimento da outra, os estudos revelaram que os CS têm eficácia analgésica similar aos AINEs (FR A). Não foram encontrados estudos relativos aos corticoides versus colchicina. No entanto, os CS apresentaram superioridade relativamente aos AINES no que respeita à segurança, por apresentarem menos efeitos adversos gastrointestinais a curto prazo (FR A). Assim sendo, são uma opção de primeira linha eficaz e segura no tratamento da AGA e estão mais indicados nos doentes que 


\begin{tabular}{|c|c|c|c|c|}
\hline Referência & População & Intervenção & Resultados & NE \\
\hline Zhang, et al. (2014) & $\begin{array}{l}\text { Pacientes com AGA com < } 24 \\
\text { horas de evolução e pelo menos } \\
\text { dor de intensidade moderada } \\
\text { ( } 2 \text { em } 5 \text { na Escala de Likert) } \\
n=60(1: 1)\end{array}$ & $\begin{array}{l}\text { Betametasona } 7 \mathrm{mg} \text { IM TU vs } \\
\text { diclofenac } 75 \mathrm{mg} \text { bid, } 7 \text { dias }\end{array}$ & $\begin{array}{l}\text { Intensidade da dor: } \\
\text { betametasona revelou-se } \\
\text { superior ao diclofenac em dia } \\
1-3(p<0,05) \text { e eficácia } \\
\text { comparável em dia } 4-7(p>0,05) \text {. } \\
\text { Não se verificaram diferenças } \\
\text { significativas no dia } 7(p>0,05) \text {. } \\
\text { Efeitos adversos: betametasona } \\
(13,3 \%) \text { vs diclofenac }(26,7 \%) \text {. }\end{array}$ & 2 \\
\hline Xu, et al. (2016) & $\begin{array}{l}\text { Pacientes com AGA com }<72 \\
\text { horas de evolução e pelo menos } \\
\text { dor de intensidade moderada } \\
\text { ( } 2 \text { em } 5 \text { numa escala de Likert) } \\
n=113\end{array}$ & $\begin{array}{l}\text { Prednisolona } 35 \mathrm{mg}(n=33) \text {, } \\
\text { etoricoxib } 120 \mathrm{mg}(n=44) \text { ou } \\
\text { indometacina } 50 \mathrm{mg} \text { tid }(n=36) \text {. }\end{array}$ & $\begin{array}{l}\text { Intensidade da dor: a } \\
\text { prednisolona oral, o etoricoxib } \\
\text { e a indometacina foram } \\
\text { similarmente eficazes durante } \\
\text { quatro dias ( } p>0,05) \text {. } \\
\text { Efeitos adversos: indometacina } \\
\text { ( } 30,6 \%) \text {, prednisolona }(6,1 \%) \text { e } \\
\text { etoricoxib }(6,8 \%)(p=0,003) \text {. }\end{array}$ & 2 \\
\hline
\end{tabular}

Legenda: $\mathrm{AGA}$ = artrite gotosa aguda; $\mathrm{IM}$ = intramuscular; $\mathrm{TU}$ = toma única; bid = duas vezes ao dia; tid = três vezes ao dia.

apresentam doenças gastrointestinais (FR A) e/ou insuficiência renal (FR C). Os COXIBs devem ser considerados em pacientes com médio ou alto risco gastrointestinal, dependendo do tipo de paciente (FR B).

É de notar que os critérios para o diagnóstico da AGA não são uniformes nos diversos estudos, ora baseando-se em critérios clínicos ora no líquido sinovial intra-articular. Para além disso, a identificação intra-articular de uratos não reflete a prática real do diagnóstico da AGA na maioria dos serviços de urgência ou consultas médicas. Na realização desta revisão, os autores puderam ainda constatar que a maior parte dos estudos utiliza diferentes outcomes para a mensuração dos resultados e que muitas NOC apresentam recomendações complexas, com diferentes graus de evidência, o que dificulta a atribuição de conclusões congruentes.

Desta forma, a escolha do agente para o tratamento da AGA deve ser determinada com base na preferência do doente, resposta prévia à terapêutica farmacológica em situação de crise, comorbilidades associadas e no número e tipo de articulações envolvidas (FR C). Não existem estudos suficientes para se puder afirmar que, para além de mais seguros, os CS são a terapêutica preferencial em relação às terapêuticas clássicas, independentemente do perfil do doente. Seria importante que os ensaios clínicos fossem uniformes relativamente à dosagem e tempo de duração dos fármacos incluídos, de forma a diminuir vieses e a permitir uma recomendação consensual das posologias adequadas para cada fármaco.

\section{REFERÊNCIAS BIBLIOGRÁFICAS}

1. Richette P, Doherty M, Pascual E, Barskova V, Becce F, Castaneda-Sanabria J, et al. 2016 updated EULAR evidence-based recommendations for the management of gout. Ann Rheum Dis. 2017;76(1):29-42.

2. Araújo F, Cordeiro I, Teixeira F, Rovisco J, Ramiro S, Mourão AF, et al. Portuguese recommendations for the diagnosis and management of gout. Acta Reumatol Port. 2014;39(2):158-71.

3. Janssens HJ, Lucassen PL, Van de Laar FA, Janssen M, Van de Lisdonk EH. Systemic corticosteroids for acute gout. Cochrane Database Syst Rev. 2008;(2):CD005521.

4. Ebell MH, Siwek J, Weiss BD, Woolf SH, Susman J, Ewigman B, et al. Strength of recommendation taxonomy (SORT): a patient-centered approach to grading evidence in the medical literature. J Am Board Fam Pract. 2004;17(1):59-67.

5. Khanna D, Khanna PP, Fitzgerald JD, Singh MK, Bae S, Neogi T, et al. 2012 American College of Rheumatology guidelines for management of gout. Part 2: therapy and antiinflammatory prophylaxis of acute gouty arthritis. Arthritis Care Res (Hoboken). 2012;64(10):1447-61.

6. Graf SW, Whittle SL, Wechalekar MD, Moi JH, Barrett C, Hill CL, et al. 
Australian and New Zealand recommendations for the diagnosis and management of gout: integrating systematic literature review and expert opinion in the 3e Initiative. Int J Rheum Dis. 2015;18(3):341-51.

7. Sivera F, Andrés M, Carmona L, Kydd AS, Moi J, Seth R, et al. Multinational evidence-based recommendations for the diagnosis and management of gout: integrating systematic literature review and expert opinion of a broad panel of rheumatologists in the $3 e$ initiative. Ann Rheum Dis. 2014;73(2):328-35.

8. Hui M, Carr A, Cameron S, Davenport G, Doherty M, Forrester H, et al. The British Society for Rheumatology guideline for the management of gout. Rheumatology. 2017;56(7):e1-e20.

9. Pérez-Ruiz F. Clinical practice guidelines for management of gout [Internet]. Madrid: Spanish Society of Rheumatology; 2013.Available from: https://www.guidelinecentral.com/summaries/clinical-practice-guidelines-for-management-of-gout/\#section-society

10. Qaseem A, Harris RP, Forciea MA. Management of acute and recurrent gout: a clinical practice guideline from the American College of Physicians. Ann Intern Med. 2017;166(1):58-68

11. Underwood M. Gout. BMJ Clin Evid. 2011 May 17;2011.

12. Wechalekar MD, Vinik O, Moi JH, Sivera F, van Echteld IA, van Durme $C$, et al. The efficacy and safety of treatments for acute gout: results from a series of systematic literature reviews including Cochrane reviews on intraarticular glucocorticoids, colchicine, nonsteroidal antiinflammatory drugs, and interleukin-1 inhibitors. J Rheumatol Suppl. 2014;92:15-25.

13. Shekelle PG, FitzGerald J, Newberry SJ, Motala A, O'Hanlon CE, Okunogbe A, et al. Management of gout [Internet]. Rockville: Agency for Healthcare Research and Quality; 2016. Available from: https://www. ncbi.nlm.nih.gov/books/NBK356141/
14. Rainer TH, Cheng CH, Janssens HJ, Man CY, Tam LS, Choi YF, et al. Oral prednisolone in the treatment of acute gout: a pragmatic, multicenter, double-blind, randomized trial. Ann Intern Med. 2016;164(7):46471.

15. Shekelle PG, Newberry SJ, FitzGerald JD, Motala A, O'Hanlon CE, Tariq $A$, et al. Management of gout: a systematic review in support of an American College of Physicians Clinical Practice Guideline. Ann Intern Med. 2017; 166(1):37-51.

16. Zhang YK, Yang H, Zhang JY, Song LJ, Fan YC. Comparison of intramuscular compound betamethasone and oral diclofenac sodium in the treatment of acute attacks of gout. Int J Clin Pract. 2014;68(5):633-8.

17. Xu L, Liu S, Guan M, Xue Y. Comparison of prednisolone, etoricoxib, and indomethacin in treatment of acute gouty arthritis: an open-label, randomized, controlled trial. Med Sci Monit. 2016;22:810-7.

\section{CONFLITOS DE INTERESSES}

Os autores declaram não ter quaisquer conflitos de interesse.

\section{FINANCIAMENTO DO TRABALHO}

Os autores declaram que o trabalho relatado neste manuscrito não foi objeto de qualquer tipo de financiamento externo.

\section{ENDEREÇO PARA CORRESPONDÊNCIA}

Ana Margarida Costa

E-mail: margarida.c.costa@hotmail.com

https://orcid.org/0000-0002-3403-583X

Recebido em 23-12-2017

Aceite para publicação em 19-09-2018

\section{ABSTRACT}

USE OF SYSTEMIC CORTICOSTEROIDS INTHE TREATMENT OF ACUTE GOUTY ARTHRITIS:WHAT ISTHE EVIDENCE? Aim: To evaluate the efficacy and safety of systemic corticosteroids (SC) in the treatment of acute gouty arthritis (AGA) and its benefits to classic treatment namely colchicine and nonsteroidal anti-inflammatory drugs (NSAIDs).

Data Sources: MEDLINE and evidence-based medicine sites.

Review methods: Clinical guidelines (NOC), systematic reviews (RS), randomized controlled trials (RCT) and meta-analyses (MA), published between June 2007 and June 2017, using the MeSH terms glucocorticoids, gout suppressants, adrenal cortex hormones, acute disease, gout, and hyperuricemia. To assess the level of evidence, the American Family Physician's Strenght of Recommendation Taxonomy (SORT) was applied.

Results: Of a total of 731 articles, eight NOC, two RS and two RCT were selected. NOC report an improvement in the symptomatology of AGA with the use of SC, NSAIDs or colchicine, differing in recommendations for the most appropriate first-line therapy. If on the one hand, some studies mention a more significant improvement of the symptoms with the use of SC, especially in the first days of treatment, on the other hand, they mention them as effective as the classic treatment. In general, all of them report more side effects in NSAID-treated patients compared to SC.

Conclusions: SC, NSAIDs, and colchicine are all effective in the therapeutic management of AGA. However, SC is superior to NSAIDs in terms of safety. Thus, the choice of agent for AGA treatment should be determined based on the patient's profile.

Keywords: Gout; Glucocorticoids. 